\title{
Análisis químico, microbiológico y sensorial de helados de mora (Rubus glaucus Benth) a base de leche de chocho (Lupinus mutabilis Sweet).
}

Chemical, microbiological and sensory analysis of blackberry ice creams

(Rubus glaucus Benth) based on lupine milk (Lupinus mutabilis Sweet).

Ronald Mauricio Zurita Gallegos. ${ }^{1}$, Byron Adrián Herrera Chávez. ${ }^{2}$ \& Jessica Alexandra Robalino Vallejo. ${ }^{3}$

Recibido: 10-05-2020 / Revisado: 15-06-2020 /Aceptado: 01-07-2020/ Publicado: 25-08-2020

\begin{abstract}
.
DOI: https://doi.org/10.33262/cienciadigital.v4i3.1443

A chemical, microbiological and sensory analysis on blackberry ice creams (Rubus glaucus Benth) was carried out, based on lupine milk (Lupinus mutabilis Sweet). A DBCA experimental design with three treatments and three repetitions was applied for lupine milk and blackberry ice creams. The information was analyzed in the SPSS program, using ANOVA and Duncan's test for protein, fat, total solids and ashes contents. A test was implemented with 36 tasters as a study group, where a verbal hedonic scale was used. Lupine milk from treatment T3 (LHC3) was considered the best treatment for making ice cream with $4.17 \%$ protein, $2.18 \%$ fat, $6.77 \%$ total solids, $0.20 \%$ ashes. For blackberry ice cream, the most accepted treatment was T1 (HLM1) with $6.21 \%$ protein, $2.20 \%$ fat, $17.84 \%$ total solids, $0.58 \%$ ashes being suitable for consumption.
\end{abstract}

Keywords: Sensory analysis, ice cream, lupine, blackberry.

\footnotetext{
${ }^{1}$ Escuela Superior Politécnica de Chimborazo, Facultad Salud Pública, Carrera de Gastronomía. Riobamba, Ecuador.ronald.zurita@espoch.edu.ec

${ }^{2}$ Universidad Nacional de Chimborazo, Facultad de Ingeniería, Carrera de Ingeniería Agroindustrial.

Riobamba, Ecuador. bherrera@unach.edu.ec

${ }^{3}$ Escuela Superior Politécnica de Chimborazo, Facultad Salud Pública, Carrera de Gastronomía. Riobamba, Ecuador.jrobalino@espoch.edu.ec
} 


\section{Resumen.}

Se realizó un análisis químico, microbiológico y sensorial de los helados de mora (Rubus glaucus Benth) a base de leche de chocho (Lupinus mutabilis Sweet). Se aplicó un diseño experimental DBCA con tres tratamientos y tres repeticiones para la leche de chocho y los helados de mora. La información fue analizada en el programa SPSS, mediante ANOVA y la prueba de Duncan para los contenidos de proteína, grasa, sólidos totales y cenizas. Se realizó un test con un grupo de estudio de 36 degustadores, donde se utilizó una escala hedónica verbal. La leche de chocho del tratamiento $\mathrm{T} 3$ (LHC3) fue considerado como el mejor tratamiento para la elaboración del helado con proteína $4.17 \%$, grasa $2.18 \%$, sólidos totales $6.77 \%$, cenizas $0.20 \%$. Para el helado de mora, el tratamiento más aceptado fue T 1 (HLM1) con proteína $6.21 \%$, grasa $2.20 \%$, sólidos totales $17.84 \%$, cenizas $0.58 \%$ siendo aptos para el consumo.

Palabras claves: Sensorial, helados, chocho, mora.

\section{Introducción.}

El síndrome metabólico (SM) es un conjunto de anormalidades consideradas como un factor de riesgo para desarrollar enfermedad cardiovascular y diabetes. (Robles, 2013). El consumo de ácidos grasos trans se relaciona con un incremento del riesgo de sufrir una enfermedad cardiovascular, debido a su efecto sobre las lipoproteínas plasmáticas (Willett, y otros, 1993).

Sin embargo, existen otros alimentos que pueden contribuir a la ingesta de ácidos grasos trans en la dieta de determinados grupos de población. Este es el caso del helado, que es un producto de amplio consumo en ciertas épocas del año, y de gran aceptación entre la población infantil. Entre sus ingredientes básicos se puede encontrar la leche o grasa láctea (helados convencionales), o bien pueden ser productos modificados preparados con grasas vegetales o aceites vegetales parcialmente hidrogenados. Por esta razón este tipo de productos deben ser muy bien considerados en las dietas alimenticias (Aro, 1998)

A través de un estudio realizado en España, varios helados comercializados de forma masiva fueron analizados en su contenido de ácidos grasos trans. La mayor parte de las muestras analizadas presentaron un valor medio del $68,1 \%$ de ácidos grasos trans, seguido de un 21,1\% de grasas monoinsaturadas y un 5,1\% de grasas poliinsaturadas (Griguol, Vicario, \& León, 2003).

Actualmente, los organismos internacionales y los consumidores adoptaron estrategias para lograr cambios actitudinales orientados hacia una alimentación saludable. En respuesta a esto, la tecnología alimentaria ha generado innovaciones e ingredientes de alto valor agregado, aplicables a alimentos funcionales (Britos, 2007). 
Tarwi o chocho es una leguminosa rica en proteínas y grasas, razón por la cual, se debería considerar como un alimento importante para formar parte de la dieta alimentaria. Su contenido proteico es incluso superior al de la soya y su contenido en grasa es similar (Castañeda Castañeda, Manrique M., Gamarra Castillo, \& Muñoz Jáuregui, 2008). Las harinas de lupinos son añadidas por su valor nutritivo (alta relación proteína-eficiencia) y también por proporcionar propiedades funcionales en productos de panadería y pastelería, en concentrados proteicos y en otros productos industriales. Esto resulta en una interesante forma de uso en la elaboración de leche sin lactosa (Castañeda Castañeda, Manrique M., Gamarra Castillo, \& Muñoz Jáuregui, 2008).

Las frutas poseen variedad de vitaminas y antioxidantes por ejemplo la mora, fruta de mayor contenido de compuestos fenólicos en comparación con las otras frutas debido a la gran cantidad en flavonoides que hacen parte de este grupo de compuestos (Ligia Rodríguez, 2010). Los flavonoides son compuestos fenólicos con una alta capacidad antioxidante que están presentes en la mayoría de las plantas, especialmente en las frutas y las hortalizas. Su actividad antioxidante ha atraído fuertemente la atención de las industrias de procesamiento de alimentos y de las compañías de pigmentos, cosméticas y farmacéuticas (Ochoa \& Ayala, 2004).

En los últimos años se ha puesto de manifiesto su importancia como antioxidante natural y su papel beneficioso, mediante su administración en la dieta, en la prevención de enfermedades cardiovasculares y algunos tipos de cáncer actuando a diferentes niveles dentro del proceso de inducción y proliferación de los tumores (Obdulio Benavente-García, 1997).

La tendencia actual es el uso de ingredientes integrales, naturales y en la reducción el aceite palma además de los aditivos como saborizantes artificiales, la reducción de grasas trans y aceites hidrogenados en los helados, es una alternativa para producir helados elaborados con leches vegetales como el tarwi o chocho que aporta con valores altamente nutricionales y frutas las cuales aportan valores de antioxidantes considerables.

Por otro lado el Análisis químico, microbiológico y sensorial de los tratamientos de helados de mora (ubus glaucus Benth) a base de leche de chocho (Lupinus mutabilis Sweet) tipo sherbet, se presentan como una interesante alternativa aportando en la tendencia actual de elaboración de helados para disminuir el uso de grasas trans.

Se obtuvo una leche de chocho (Lupinus mutabilis Sweet) con excelentes características nutricionales, aplicable en la elaboración de helados de frutas andinas mora (Rubus glaucus

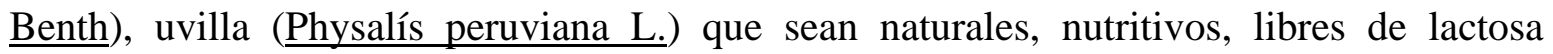
considerado un helado tipo Sorbete o Sherbet según las normas NTE INEN 706 2005, con un buen grado de aceptabilidad. 


\section{Metodologia.}

La primera parte de la investigación se orientó a la seleccionó del chocho y la mora como ingredientes para la elaboración del helado adquiriéndolos en un mercado de la ciudad de Riobamba y considerando los parámetros pertinentes para la realización de la investigación los ingredientes adquiridos fueron llevados a los laboratorios de la Escuela de Gastronomía de la Facultad de Salud pública de la Escuela Superior Politécnica de Chimborazo, cantón Riobamba, Provincia de Chimborazo, Ecuador.

La investigación tuvo dos fases, la primera fue la obtención de la materia prima en donde se utilizó un diseño experimental (DBCA) con tres tratamientos y tres repeticiones para la leche. Para la segunda fase de la elaboración del helado de mora a base de leche de chocho se utilizó tres tratamientos con tres repeticiones, teniendo un total de 18 unidades experimentales.

Leche de chocho: se seleccionó el mejor grano de chocho además se lavó y se blanqueó en agua a $96^{\circ} \mathrm{C}$ por 2 min. se pesaron los ingredientes previo a la realización de los tratamientos planteados, posteriormente se escaldo a $96^{\circ} \mathrm{C}$ por 3 min. y se licuó cada una de las mezclas por $1 \mathrm{~min}$. a $28^{\circ} \mathrm{C}$ además se cernió para luego pasteurizar a $70{ }^{\circ} \mathrm{C}$ por $15 \mathrm{~min}$. Se enfrió a 4 ${ }^{\circ} \mathrm{C}$ la mezcla, envasándola para su análisis de proteína, grasas, sólidos totales y cenizas de los tratamientos realizados.

Helado de mora a base de leche de chocho: Una vez seleccionada la leche de chocho con mayor valor nutricional se licuó con la mora y adición de azúcar se licuó y cernió para pasteurizar la mezcla a $70^{\circ} \mathrm{C}$ por $15 \mathrm{~min}$, después se enfrió hasta llegar a una temperatura de $0^{\circ} \mathrm{C}$ en donde se procedió a madurar la mezcla a $0^{\circ} \mathrm{C}$ aproximadamente por $24 \mathrm{~h}$.

La homogenización y congelación se la realizó batiendo hasta que se enfríe y se rompan todos los cristales hasta tener una pasta de hielo a $-6^{\circ} \mathrm{C}$ por $2 \mathrm{~h}$. donde se colocó en envases esterilizados, sellados y etiquetando las muestras a $-6^{\circ} \mathrm{C}$. para la determinación de proteína, grasas, sólidos totales, cenizas, así como los parámetros microbiológicos como Coliformes, Eschericha Coli, Aerobios Mésofilos, Mohos y Levaduras, Salmonella, Staphylococcus, Listeria Monocytogenes.

Una vez realizado todos los análisis previos se continuo con una evaluación señorial por medio de una prueba de aceptabilidad a los helados de los distintos tratamientos aplicada con una escala hedónica verbal a 36 estudiantes de la carrera de gastronomía para determinar la aceptabilidad de los helados.

La estadística se aplicada a los datos obtenidos en los diferentes factores fue un ANOVA y para determinar cambios significativos y para mayor certeza de esos cambios de aplico una de post hoc en base a la prueba de Duncan al $0.05 \%$ utilizando el programa SPSS. Para la evaluación sensorial mediante un test de aceptabilidad con escala hedónica verbal se utilizó una estadística descriptiva dándole valores cuantitativos. 


\section{Resultados}

\section{Leche de chocho}

Tabla 1 Características químicas de la leche de chocho

\begin{tabular}{cccccc}
\hline Indicadores & T 1 (TLCH1) & T 2 (TLCH2) & T 3 (TLCH3) & cv \% & Media \\
\hline Proteína & $3.75 \mathrm{c}$ & $3.93 \mathrm{~b}$ & $4.17 \mathrm{a}$ & 5.07 & 3.95 \\
Grasa & $2.04 \mathrm{a}$ & $2.13 \mathrm{a}$ & $2.18 \mathrm{a}$ & 11.79 & 2.12 \\
Sólidos Totales & $6.46 \mathrm{a}$ & $6.46 \mathrm{a}$ & $6.77 \mathrm{a}$ & 7.77 & 6.56 \\
Cenizas & $0.19 \mathrm{a}$ & $0.20 \mathrm{a}$ & $0.20 \mathrm{a}$ & 4 & 0.20 \\
\hline
\end{tabular}

Nota: $<0,05$ (Duncan. 1955), letras iguales no difieren significativamente.

Fuente: Elaboración propia

\section{Contenido de proteína de la leche de chocho.}

El contenido de Proteína registró un valor de $3.95 \%$ con un coeficiente de variación de 5.07 $\%$ al analizar los resultados se encontraron diferencias significativas entre los diferentes tratamientos. En la Tabla 1 aplicando la prueba de Duncan se observa que existen diferencias significativas entre los 3 tratamientos donde el tratamiento T1 (LCH1) difiere significativamente del tratamiento T2 (LCH2) y este a su vez con el tratamiento T3 (LCH3), tomando en cuenta que el tratamiento T3 tiene más contenido de proteína con $4.17 \%$.

\section{Contenido de grasa de la leche de chocho}

El contenido de Grasa registró una media de $2.12 \%$ con un coeficiente de variación de 11.79 $\%$ al analizar los resultados no se encontraron diferencias significativas entre los diferentes tratamientos. Se observa en la Tabla 1 aplicando la prueba de Duncan los contenidos de grasa no difieren significativamente entre los 3 tratamientos, siendo el tratamiento T 3 (LCH3) que contiene $2.18 \%$ de grasa.

\section{Contenido de sólidos totales de la leche de chocho}

El contenido de Sólidos Totales registró un valor de $6.56 \%$ con un coeficiente de variación de $7.77 \%$ al analizar los resultados no se encontraron diferencias significativas entre los diferentes tratamientos. En la Tabla 1 se observa que no hay diferencias significativas entre los 3 tratamientos aplicando la prueba de Duncan siendo el tratamiento T3 (LCH3) que registro $6.77 \%$ de sólidos totales.

\section{Contenido de cenizas de la leche de chocho}

El contenido de Ceniza registró un valor de $0.20 \%$ con un coeficiente de variación de $4 \%$ al analizar los resultados no se encontraron diferencias significativas entre los diferentes tratamientos. En la Tabla 1 aplicando la prueba de Duncan se observa que no hay diferencias 
significativas entre los 3 tratamientos siendo el tratamiento T3 (LCH3) con un contenido de $.20 \%$ de cenizas.

\section{Helados de Mora a Base de Leche de Chocho}

Tabla 2 Contenidos nutricionales del helado de mora a base de leche de chocho

\begin{tabular}{ccccccc}
\hline Indicadores & T 1 (HLM1) & T 2 (HLM2) & T 3 (HLM3) & cv \% & Media \\
\hline Proteína & $6.21 \mathrm{a}$ & $6.29 \mathrm{a}$ & $6.76 \mathrm{a}$ & 7.01 & 6.42 \\
Grasa & $2.20 \mathrm{a}$ & $1.07 \mathrm{~b}$ & $2.10 \mathrm{a}$ & 31.84 & 1.79 \\
Sólidos Totales & $17.84 \mathrm{a}$ & $16.54 \mathrm{a}$ & $18.24 \mathrm{a}$ & 8.06 & 17.54 \\
Cenizas & $0.58 \mathrm{a}$ & $0.31 \mathrm{a}$ & $0.32 \mathrm{a}$ & 52.5 & 0.40 \\
\hline
\end{tabular}

Nota: $<0,05$ (Duncan. 1955), letras iguales no difieren significativamente.

Fuente: Elaboración propia

\section{Contenido de proteína del helado de mora a base de leche de chocho}

El contenido de Proteína registró un valor de $6.42 \%$ con un coeficiente de variación de 7.01 $\%$ al analizar los resultados no se encontraron diferencias significativas entre los diferentes tratamientos. En la (Tabla 2) mediante la prueba de Duncan no hay diferencias significativas siendo el tratamiento T3 (HLM3) que registro 6,76 \% en el contenido de proteína.

\section{Contenido de grasa del helado de mora a base de leche de chocho}

El contenido de Grasa registró un valor de $1.79 \%$ con un coeficiente de variación de 31.84 $\%$ al analizar los resultados se encontraron diferencias significativas entre los diferentes tratamientos. En la (Tabla 2) aplicando la prueba de Duncan hay diferencias significativas en el contenido de grasa siendo el tratamiento T2 (HLM2) que difiere del tratamiento T3 (HLM3) y T1 (HLM1) siendo este último tratamiento el que tiene $2.20 \%$ en relación a los otros tratamientos.

\section{Contenido de Sólidos Totales del helado de mora a base de leche de chocho}

El contenido de Sólidos Totales registró un valor de $17.54 \%$ con un coeficiente de variación de $8.06 \%$ al analizar los resultados no se encontraron diferencias significativas entre los diferentes tratamientos. En la Tabla 2 mediante la prueba de Duncan no hay diferencias significativas siendo el tratamiento T3 (HLM3) el que tiene un valor de 18,24 \% de Sólidos Totales.

\section{Contenido de cenizas del helado de mora a base de leche de chocho}

El contenido de Ceniza registró un valor de $0.40 \%$ con un coeficiente de variación de 52.15 $\%$ al analizar los resultados no se encontraron diferencias significativas entre los diferentes tratamientos. En la Tabla 2 mediante la prueba de Duncan no hay diferencias significativas siendo el tratamiento T 1 (HLM1) el que tiene un valor de 0,58 \% de Cenizas. 
Análisis microbiológicos de los helados de mora a base de leche de chocho

Tabla 3 Resultados de pruebas microbiológicas

\begin{tabular}{|c|c|c|c|c|c|c|c|c|c|c|}
\hline Indicadores & 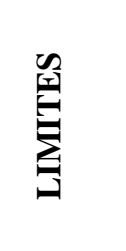 & 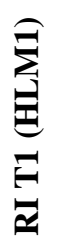 & 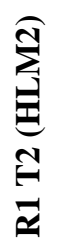 & 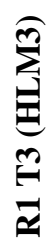 & 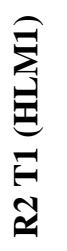 & 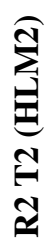 & 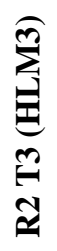 & 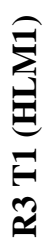 & 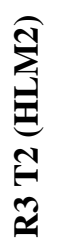 & 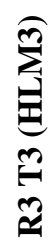 \\
\hline Coliformes Totales UFC/g & 200 & - & 100 & - & - & - & - & - & - & - \\
\hline Eschericha Coli UFC/g & - & - & - & - & - & - & - & - & - & - \\
\hline Aerobios Mésofilos UFC/g & 100000 & 70 & - & - & - & - & - & - & - & - \\
\hline Mohos y Levaduras UFC/g & - & - & - & - & - & - & - & - & - & - \\
\hline Salmonella UFC/25g & 100 & - & - & - & - & - & - & - & - & - \\
\hline Staphylococcus UFC/g & - & 60 & - & - & - & - & - & - & - & - \\
\hline Listeria Monocytogenes UFC/25g & - & & - & - & - & - & - & - & - & - \\
\hline
\end{tabular}

Nota: el Símbolo (-) representa ausencia de patógeno

\section{Coliformes Totales}

En las pruebas microbiológicas Tabla 3 se determinó ausencia de Coliformes Totales.

\section{Eschericha Coli}

En los resultados microbiológicos de Escherichia Coli se declaró ausencia (Tabla 3)

\section{Aerobios mesófilos}

En los análisis microbiológicos en la repetición R1 HLM1 identificó (70UFC/g) (Tabla 3), encontrándose en los rangos permitidos por las normas INEN.

\section{Mohos y Levaduras}

En los resultados obtenidos se determinó ausencia de Mohos y levaduras (Tabla 3).

\section{Salmonella}

Los análisis microbiológicos de Salmonella declararon ausencia de los mismos. (Tabla 3)

\section{Aerobios mesófilos}

En los análisis microbiológicos en la repetición R1 HLM1 identificó (70 UFC/g) (Tabla 3), encontrándose en los rangos permitidos por las normas INEN.

\section{Mohos y Levaduras}

En los resultados obtenidos se determinó ausencia de Mohos y levaduras (Tabla 3). 


\section{Salmonella}

Los análisis microbiológicos de Salmonella declararon ausencia de los mismos. (Tabla 3)

\section{Stafhylococcus}

Se identificó presencia de stafhylococuccus (Tabla 3), en la repetición R1 HLM1 con (60 UFC/g), encontrándose en los rangos establecidas por las normas INEN.

\section{Listeria Monocytogenes}

Los análisis microbiológicos de Listeria Monocytogenes declararon ausencia (Tabla 3).

\section{Test de Aceptabilidad}

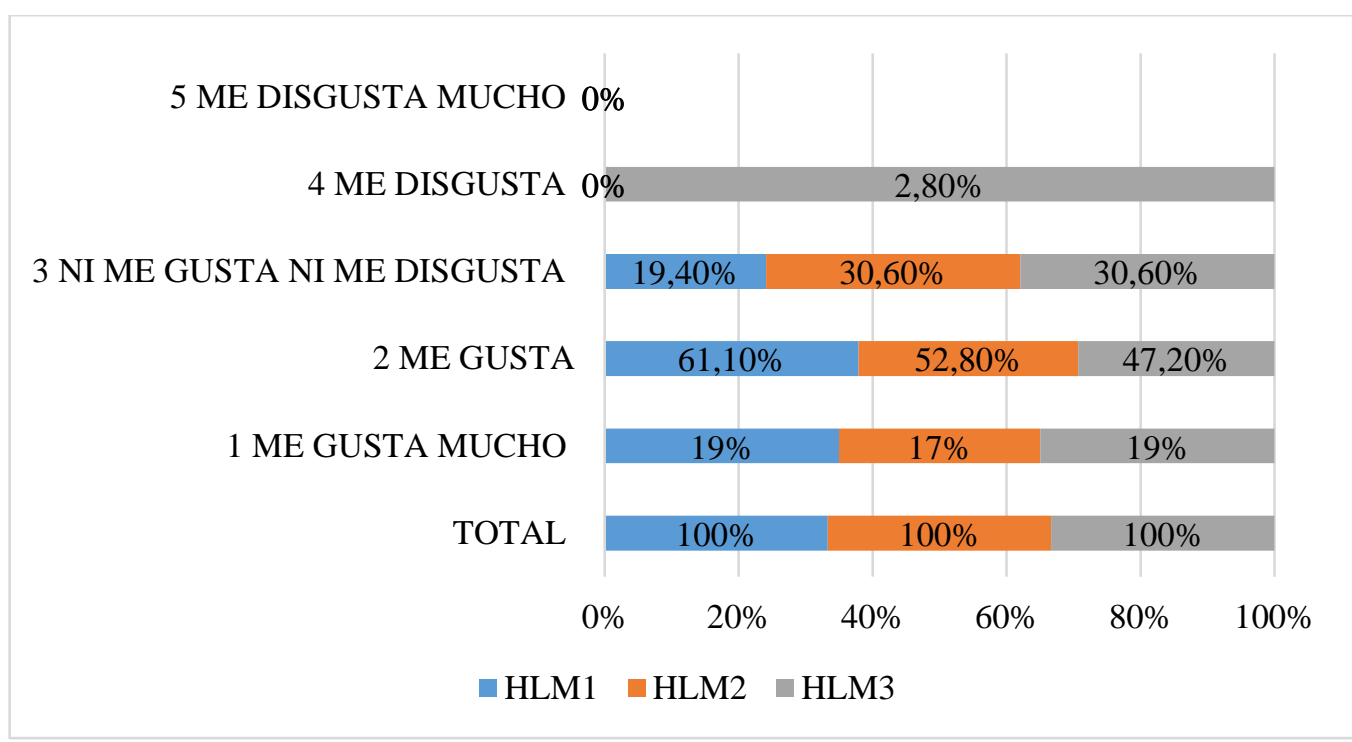

Figura 1 Test de aceptabilidad de helados de mora a base de leche de chocho Fuente: Elaboración propia

\section{Análisis del test de aceptabilidad de los helados de mora a base de leche de chocho}

En el gráfico N1 está la representación por porcentaje obtenidos de una evaluación sensorial mediante escala hedónica verbal donde se puede determinar la aceptabilidad del producto final, como se puede observar el Tratamiento T1 (HLM1) contiene $61.10 \%$ en la escala de me gusta, por su formulación que contienen mayor porcentaje de mora con respecto a la leche de chocho sobresaliendo de los demás tratamientos por su olor y color rojo intenso característico de la fruta con un sabor dulce y una textura cremosa, el tratamiento T2 (HLM2) con $52.89 \%$ seguido del tratamiento T3 (HLM3) con $47.20 \%$ de aceptabilidad. Se puede atribuir a que en mayor porcentaje de mora es más agradable el helado y a medida que disminuye la fruta y aumenta la leche de chocho disminuye la aceptabilidad del helado por mayor presencia del sabor de la leche de chocho. 


\section{Discusión de resultados.}

La proteína encontrada en la leche de chocho T (LCH3) es de $4.17 \%$ siendo este tratamiento el mejor con alto valor para usar en la elaboración de helados de mora a base de leche de chocho, en relación a la leche de chocho según (E. Villacrés 2006) La proteína de leche de chocho encontrada en su investigación es de $3.5 \%$.

En la investigación de (E. Villacrés 2006) la leche de chocho aporta $1.6 \%$ de grasa, mientras que en la presente investigación el tratamiento T 3 (LCH3) con $2.18 \%$

La contenido de sólidos encontrada en esta leche de chocho es de alto valor nutritivo en relación a los otros tratamientos ya que es de $6.77 \%$ del tratamiento T 3 (LCH3) tal vez se deba a que el diámetro del lienzo para tamizar era muy pequeño y atravesaron partículas muy pequeñas de sólidos a través de él; según (Luis Dyner1 2015) El mayor porcentaje de sólidos totales (10\%) se halló en la muestra obtenida sin remojo y con filtración (M1); esto se refiere a una bebida artesanal a base de almendras; mientras que en la investigación de leche de chocho de (Luis Dyner1 2015) se determinó un 12.54 \% de sólidos totales.

La leche de almendras presenta mayor porcentaje de cenizas, es decir mayor contenido de minerales; con $0.30 \%$ (Luis Dyner1 2015), entretanto el contenido cenizas encontrada en la presente investigación el tratamiento T 3 (LCH3) la leche de chocho tiene un valor de 0.20 $\%$ siendo un porcentaje moderado de cenizas.

Un helado de tomate elaborado a base de leche de soya "contiene 2,6 g. en 60 g. en porción de helado" (Daniela Berestan 2010); según (Ibarra Hernández B. 2016) “el helado se elaboró por un proceso artesanal empleando como ingredientes leche de soya, harina de amaranto, azúcar, huevo, vainilla y grenetina. Con un contenido de $12.5 \%$ de proteína"; hay que tener en cuenta que los helados elaborados a base de leche de chocho no fueron fortificados con ningún tipo de proteína de origen animal, siendo así la proteína encontrada en el helado de mora a base de leche de chocho con $6,76 \%$ en el tratamiento T 3 (HLM3).

El producto tipo helado a base de soya y amaranto Según (Ibarra Hernández B. 2016) “el helado desarrollado presentó un contenido proteico 3.5 veces mayor que el producto comercial y aproximadamente 5 veces menor de grasa"; se puede observar en la tabla de composición proximal del helado desarrollado contiene $2.3 \%$ de grasa; mientras que la grasa 
encontrada en el Helado de mora a base de leche de chocho es de $2.20 \%$ es del tratamiento

T 1 (HLM1).

La investigación llamada Utilización de Leche de Soya en Helado, la composición del helado a elaborar fue la siguiente: $7 \%$ de grasa, $7 \%$ de sólidos no grasos (leche y soya) y 31,6 \% de sólidos totales (mínimos). Según (Tamara Rodríguez* 2010) mientras que los sólidos totales encontrada en la presente investigación del helado de mora a base de leche de chocho del el tratamiento T 3 (HLM3) con un contenido de 18,24 \% de sólidos totales.

El contenido de cenizas encontrada en esta leche es de alto valor que es de 0,58\% que describe el tratamiento T 1 (HLM1); la composición proximal del contenido de cenizas del helado de elaboración de un producto tipo helado a base de soya (glycine max) y amaranto (amaranthus cruentus) con jugo de fruta esferificado según (Ibarra Hernández B. 2016) es de $0.5 \%$.

Se puede determinar que los helados se encuentran dentro de los parámetros microbiológicos de las normas NTE INEN 7062005

\section{Conclusiones.}

- La leche de chocho del tratamiento T3 (LCH3) se consideró como el mejor tratamiento para la elaboración del helado, siendo su composión bromatológica su proteína de $4,17 \%$, sólitdos totales $6.77 \%$, grasa $2.18 \%$ y $0.20 \%$ de cenizas.

- De los procesos de elaboración de los helados de mora a base de leche de chocho realizados con 3 tratamientos se analizaron los parámetros químicos y microbiológicos cumpliendo con las normas NTE INEN 7062005 donde clasifica como tipo Sherbet y para poder determinar cuál fue el mejor tratamiento se realizó un test de aceptabilidad con un grupo de estudio de 36 degustadores aplicando una escala hedónica verbal la cual fue analizada para determinar la aceptabilidad siendo el T1 (HLM1) del helado de mora a base de leche de chocho el mejor tratamiento obtenido en donde registro proteína $6.21 \%$, sólidos totales $17.84 \%$, grasa $2.20 \%$ y ceniza $0.58 \%$

- En la elaboración de helados de mora a base de leche de chocho se pudo determinar ausencia de microorganismo patógenos como coliformes totales, eschericha coli, salmonella, mohos y levaduras; en aerobios mésofilos y Stafhylococcus se encuentran en el rango permisible por lo cual es apto para el consumo humano.

- La elaboración de helado de leche de chocho, se constituye en una alternativa saludable, sin embargo, es necesario continuar con los procesos de investigación que permiten ir mejorando el sabor y la calidad de palatabilidad del producto con el 
propósito de promover su uso comercial, la alternabilidad de este producto podría iniciarse en restaurantes de cocina gourmet como una propuesta innovadora.

\section{Referencias Bibliográficas}

Aro, A. A.-B. (1998). Trans fatty acids in dietary fats and oils from 14 european countries: the TRANSFAIR study. 137-149.

Britos, S. (2007). Transición nutricional, obesidad y desafíos de las políticas públicas y los agronegocios. Nutrinfo.

Castañeda Castañeda, B., Manrique M., R., Gamarra Castillo, F., \& Muñoz Jáuregui. (2008). Probiótico elaborado en base a las semillas de . Acta Médica Peruana, 213.

Daniela Berestan, M. P. (2010). Helado de verduras a base de leche de soja. Invenio Revista de investigación académica, 144. Obtenido de Dialnet .

Griguol, V., Vicario, ... \& León, M. (2003). Contenido en isómeros geométricos de los ácidos grasos en helados comerciales. CSIC, 19.

Ibarra Hernández B., S. F. (2016). Investigación y Desarrollo en Ciencia y Tecnología de Alimentos. Obtenido de Investigación y Desarrollo en Ciencia y Tecnología de Alimentos: http://www.fcb.uanl.mx/IDCyTA/files/volume1/2/8/99.pdf

Ligia Rodríguez, L. L. (2010). Determinación de la composición química y actividad antioxidante en distintos estados de madurez de frutas de consumo habitual en colombia, mora (Rubus glaucus b.), maracuyá (Passiflora edulis s.), guayaba (Psidium guajava 1.) y Papayuela (Carica cundina). Revista Alimentos Hoy. I.

Luis Dyner1, M. B. (2015). Portal Regional da BVS. Obtenido de Portal Regional da BVS: http://pesquisa.bvsalud.org/portal/resource/pt/lil-771527

Obdulio Benavente-García, J. C. (1997). Uses and Properties of Citrus Flavonoids. American Chemical Society, 45 (12), pp 4505-4515. Obtenido de http://pubs.acs.org/doi/pdf/10.1021/jf970373s

Robles, J. C. (2013). Síndrome metabólico: concepto y aplicación práctica. Scielo Perú , 315.

Tamara Rodríguez*, J. C. (2010). UTILIZACIÓN DE LECHE DE SOYA EN HELADO. CIENCIA Y TECNOLOGÍA DE ALIMENTOS, 28. Obtenido de http://www.hablemosclaro.org/Repositorio/biblioteca/b_255_Leche_de_soya_en_he lado.pdf 
Villacrés, E. (2006). http://repositorio.educacionsuperior.gob.ec/handle/28000/910. (I.

Quito, Ed.) Recuperado el 2016, de

http://repositorio.educacionsuperior.gob.ec/handle/28000/910:

http://repositorio.iniap.gob.ec/bitstream/41000/298/1/iniapscbd333.pdf

Willett, W. C., Stampfer, M. J., Manson, J. E., Colditz, G. A., Speizer, F. E., Rosner, B. A., ... Hennekens, C. H. (1993). Intake of trans fatty acids and risk of coronary. 581585. 


\section{PARA CITAR EL ARTÍCULO INDEXADO.}

Zurita Gallegos, R. M., Herrera Chávez, B. A., \& Robalino Vallejo, J. A. (2020). Análisis químico, microbiológico y sensorial de helados de mora (Rubus glaucus Benth) a base de leche de chocho (Lupinus mutabilis Sweet). Ciencia Digital, 4(3), 391-403. https://doi.org/10.33262/cienciadigital.v4i3.1443

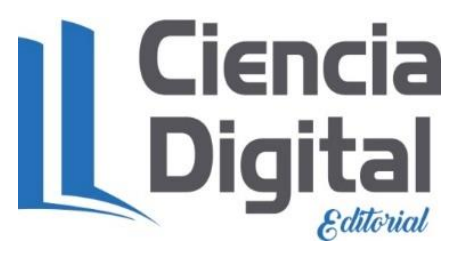

El artículo que se publica es de exclusiva responsabilidad de los autores y no necesariamente reflejan el pensamiento de la Revista Ciencia Digital.

El artículo queda en propiedad de la revista y, por tanto, su publicación parcial y/o total en otro medio tiene que ser autorizado por el director de la Revista Ciencia Digital.
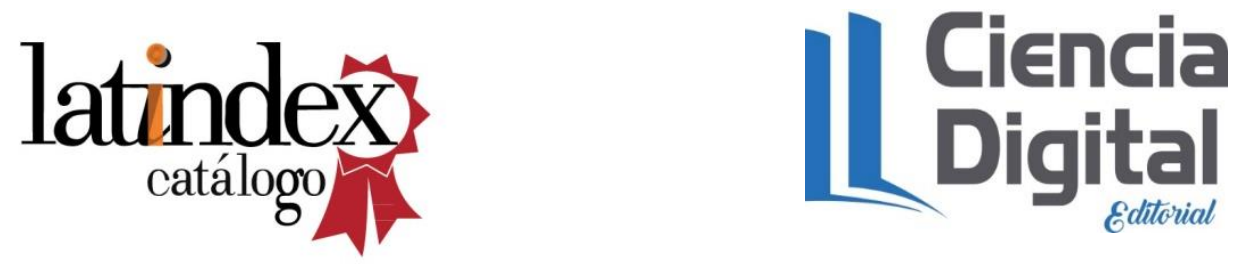